\title{
The Modification and Implementation of Campus Network Client
}

\author{
Lingfang Huang ${ }^{a}$ \\ Institute of Mathematics and Computer, Jiangxi Science \& Technology Normal University, Nanchang \\ 330009, China \\ aANAN_60@163.com
}

Keywords: Client; PPPoE; Networking Certification.

Abstract. In this paper, the campus network client certification mechanism is analyzed, replacement client is rewritten, the stability is greatly improved and the sharing function of WiFi is strengthened.

\section{Introduction}

The SRUN client adopts the PPPoE protocol in our school currently, but the client carry out certain extension to PPPoE protocol, which causes users must use the client access networks, thus prohibit users from using routers and dial directly by PPPoE. Because the beginning of design of the client is to strictly control the network usage condition, abandon the router and multi-network card network share, therefore, a series of network control module is added, network sharing is forbidden, if detect the system uses multi-network card sharing, then carry out offline processing. Although this mechanism prohibits network sharing, consequently the instability emerges in network, network sharing detecting often turns up error check to cause network disconnected.

\section{The Principle Analysis of Campus Network Client (SRUN)}

The SRUN client uses PPPoE protocol to implement network authentication, and a certain extension is done on the basis of the PPPoE protocol. The main content of extension is that encrypts PPPoE dial-up username, hide the real user name, and add escape character that the keyboard can't directly input in the real user name in order to avoid users carrying on connects certification around the client. Afterwards the the heartbeat is extended in PPPoE protocol, their heartbeats are increased based on the original heartbeats detection, the client regularly transmit heartbeat packets that contain the user information, and then the server respond specified content so as to judge the client online condition, in order to release the network resources.

\section{The Client Function Planning}

The substitute goods of SRUN client should have most of the functions of original client except "agent software detection", the preliminary plan is as follows:

Account information conversion, PPPoE dial, remember the password, disconnect network, automatic connection, information encryption, human-computer interaction, error message processing, self-startup, configuration information, help demonstration, function reset, heartbeat detection, etc.

The remember password using the account and password stored in the external memory (hard disk), in order to prevent others from finding the file to view, therefore, the information in the password file is encrypted, after encryption the file is saved. While reading the password, the content is decrypted.

When users first start the program to create a new connection called XQ_KLZF, and build configuration files, when user make the corresponding operation, configuration file also makes corresponding changes. Each time you start the program, the program has priority to deal with the transmitting parameters of main function, then reads the configuration file to determine whether or 
not the user has made automatically connected, save the password and son on, if exist, the corresponding function should be executed.

The self-startup is completed by adding Windows registry startup, the automatic connection parameters is transmitted to automatically connect.

To restore the default settings module will delete the password files, configuration files, and all the registry addition items. Disconnect the connection module will enumerate all existing connection, if connect, the network will be disconnected. PPPoE dial module adds the connection detection to determine whether the user's computer connect the Internet or not, and give a hint.

After the heartbeat detection module dial successfully, it will be operate and resident in memory, and also plays a break line detection, offline reconnection functions. Based on the point of view of saving memory, the heartbeat detection is alone as a program module, after the main program successfully dial, the heartbeat detection program are invoked by the main program, and the account information in the form of parameters are passed to the heartbeat detection module, then the main program automatically quit, release the memory of main program, heartbeat detection program runs in the background separately. When the heartbeat detection programs find network anomalous, it pulse hints, and invoke the main program, after the main program dial successfully, the heartbeat detection program is restarted.

\section{The Core Function Effect of the Client}

\subsection{Build a Phone Book Entry}

The phone book entry that used connection establish DWORD CreateDial (wchar_t *DialName) function creates, the function transmit parameters for phone book entry name. The RasSetEntryProperties function of Windows API is invoked the in this function.

This main function of function is to set up attribute of phone book entry, or set up a new phone book entry. It has six parameters, the first parameter is the LPCTSTR lpszPhonebook, this parameter is string pointer and is used to specify the path and name of the phone book, if this parameter is null, then select the default directory. Here, we ignore this parameter and set to NULL.

The second parameter is LPCTSTR lpszEntry, this parameter transmit a string pointer that represent entry name, if the entry name has already existed in the system, then modify the corresponding attributes, if it has not exist, a new entry will be created.

The third parameter LPRASENTRY LPRASENTRY is a pointer to the RASENTRY structure. This structure contains 55 members.

The fourth parameter DWORD dwEntryInfoSize represent that the RASENTRY structure size third parameter transmitted is to use RasGetEntryProperties () function.

The fifth parameter LPBYTE lpbDeviceInfo is to formulate configuration information of equipment, this parameters is not available under XP system, it must be NULL.

Sixth parameter DWORD dwDeviceInfoSize represent the size of the fifth parameter, beacuse the fifth parameter is NULL, so this parameter is empty.

Sixth parameters is transmitted, RasSetEntryProperties function is to set up a new phone book entry, if it is created successfully, the return value is zero.

\subsection{Dial}

The dial takes RasDial()function, six parameters exist in function, function prototype is as follows:

DWORD RasDial(

$$
\begin{aligned}
& \text { _In_LPRASDIALEXTENSIONS lpRasDialExtensions, } \\
& \text { _In_ LPCTSTR lpszPhonebook, } \\
& \text { _In_L LPRASDIALPARAMS lpRasDialParams, } \\
& \text { _In_ DWORD dwNotifierType, } \\
& \text { _In_LPVOID lpvNotifier, } \\
& \text { _Out_ LPHRASCONN lphRasConn } \\
& \text { ); }
\end{aligned}
$$


The first parameter is a pointer to the RASDIALEXTENSIONS structure, this structure can set some extension function of the RasDial, if you don't need these functions, it can be set to null. Here we don't need these extension features, so it is set to NULL.

The second parameter LPCTSTR lpszPhonebook is making path and file name of telephone book, because we use the default directory, thus also NULL.

The third parameter LPRASDIALPARAMS LPRASDIALPARAMS is a pointer to the RASDIALPARAMS structure. There are ten members; this structure prototype is as follows:

Typedef struct_RASDIALPARAMS \{

// the size of the structure, a specific value must be set, or the 632 error will be submitted

DWORD dwSize;

// create a phone book entry

TCHAR szEntryName [RAS_MaxEntryName + 1];

// set a prior telephone number, if szEntryName is not null, the value can be null

TCHAR szPhoneNumber [RAS_MaxPhoneNumber + 1];

// back dial phone number is null which represent s you can't back

TCHAR szCallbackNumber [RAS_MaxCallbackNumber + 1];

// dial-up used username

TCHAR szUserName [UNLEN + 1];

// dial-up used passwords

TCHAR szPassword [PWLEN + 1];

// accredited domain name is empty,which represents specified domain name is one member of the server

TCHAR szDomain [DNLEN + 1];

// the index of the initial phone book entry

DWORD dwSubEntry;

// specified value back to callback function RasDialFunc2

ULONG_PTR dwCallbackId;

// the specified value connected VPN

DWORD dwIfIndex;

\} RASDIALPARAMS, * PRASDIALPARAMS;

The corresponding information is filled to the structure, it as a parameter transmitted to the RasDial function.

The fourth parameter is DWORD dwNotifierType, this parameter is used to set the callback function type, if empty, it is ignored.

The fifth parameter is LPVOID lpvNotifier, it is used to set the window handle or the callback function to receive the event notification of RasDial function.

The last parameter LPHRASCONN LphRasConn is a pointer with HRASCONN type, the pointer is initialized to NULL, when dialing successfully, the handle that the RAS connected will be put into it.

The function can carry out dialing, if dial successfully, return value is zero, if error appears, returns the corresponding error code.

\subsection{The 3UDP Heartbeat Packets Transmission}

The heartbeat package is UDP packets with specific content, UDP packets transmit with C Socket program.

Using WSAStartup initialize WinSocket, if succeed, it returns 0.

Function prototype is:

Int WSAStartup (

WORD wVersionRequested, // show that supported highest version number

Sockets LPWSADATA LPWSADATA // a pointer to the WSADATA structure, it is used to set the details 
Use the socket (PF_INET, SOCK_DGRAM, 0) to build a datagram UDP socket, the function prototype is as follows:

Int the socket (

Int domain, // agreement domain, which indicates Socket address types, PF_INET represent use

// ipv4 addresses (32) and the combination of the port number (16)

int type, // the specified Socket type, SOCK_DGRAM represent UDP type

int protocol // specified protocol, parameters IPPROTO_UDP is UDP protocol,

// it is set to 0 , will automatically match the agreement adopted by

the second parameter

Setsockopt function is used to set the socket option, if need to broadcast form, the third parameter will be set to SO_BROADCAST, this function can be set to send and receive overtime time, specific examples are as follows:

The setsockopt (sockListener, SOL_SOCKET, SO_SNDTIMEO, (char *) \& a timeout, sizeof (int)); // set send timeout

The setsockopt (sockListener, SOL_SOCKET, SO_RCVTIMEO, (char *) \& a timeout, sizeof (int)); // set receive overtime

The function prototype:

Int setsockopt (

Int sockfd, // mark description words of one socket

Int level, // optional defined levels

Int optname, // set options needed

Const void * optval, // buffer pointer of new value pointed to storage option setting

Socklen_t optlen // the length of above parameter

After setting up the destination address and port number, the sendto, recv function can be used for sending and receiving.

If send or receive data timeout, it means that the network has been disconnected; the corresponding hint will emerge, and start the main program to redial.

After program testing and running over a period of time, the client overcome the instability of the past, the user reflect well, which not only enhances the network stability, but also strengthens the Wi-Fi sharing function.

\section{References}

[1] http://http://baike.baidu.com/view/27505.htm

[2] http://zh.wikipedia.org/wiki/PPPoE

[3] Tang Yuan, Kong Fansheng. PPPoE dial-up software implementation Under Windows [D]. [dissertation]. Zhejiang University, 2005.

[4] Tao Lan Guan XiaoHua. PPPoE protocol implementation based on Windows system [J]. Journal of China Agricultural University. 2002, 7 (6): 78-81.

[5] Mamakos, et. Al. Transmitting the PPP over Ethernet [J]. Journal of RFC 2516. 1999 (02):17.

[6] http://zh.wikipedia.org/wiki/\%E5\%BC\%82\%E6\%88\%96\%E5\%AF\%86\%E7\%A0\%81

[7] http://baike.baidu.com/view/674171.htm

[8] Hans \& Knebl, Helmut. Symmetric - key encryption. The Introduction to cryptography [M]. 2007.

[9] Brian W. Kozirenski Ernighan, Dennis M.Ritchie. The C Programming Language [M]. China Machine Press. 2004.

[10] http://MSDN Library. https://msdn.microsoft.com/zh-cn/library/

[11] Charles Petzold. Windows programming [M]. Tsinghua university press. 2010. 
[12] Xie Xiren. Computer networks [M]. Beijing. Publishing House of Electronics Industry. 2008. 